\title{
Beckett, Mahon, Carson et Le Bateau ivre:Rythme, prosodie, interprétation*
}

\section{Maryvonne Boisseau}

\section{Q OpenEdition \\ 1 Journals}

\section{Édition électronique}

URL : http://journals.openedition.org/etudesirlandaises/2003

DOI : 10.4000/etudesirlandaises.2003

ISSN : 2259-8863

\section{Éditeur}

Presses universitaires de Rennes

\section{Édition imprimée}

Date de publication : 30 décembre 2010

Pagination : 135-148

ISBN : 978-2-7535-1246-7

ISSN : 0183-973X

\section{Référence électronique}

Maryvonne Boisseau, "Beckett, Mahon, Carson et Le Bateau ivre:Rythme, prosodie, interprétation* », Études irlandaises [En ligne], 35-2 | 2010, mis en ligne le 30 décembre 2012, consulté le 19 avril 2019. URL : http://journals.openedition.org/etudesirlandaises/2003; DOI : 10.4000/etudesirlandaises.2003

Ce document a été généré automatiquement le 19 avril 2019.

(c) Presses universitaires de Rennes 


\title{
Beckett, Mahon, Carson et Le Bateau ivre:Rythme, prosodie, interprétation*
}

\author{
Maryvonne Boisseau
}

Grammaire :

1. Ensemble des règles à suivre pour parler et écrire correctement une langue.

2. Ling. étude systématique des éléments constitutifs d'une langue, qui sont réductibles à des règles et relèvent de régularités; spécialt. Étude des formes et des fonctions. Ensemble des structures et des règles qui permettent de produire tous les énoncés d'une langue et seulement ceux-ci.

2 Prosodie :

(empr. au latin, prosodia "accent tonique, quantité de syllabes", pris au grec prosôdia "chant pour accompagner la lyre», "variation dans le niveau de la voix» et "prononciation d'une syllabe accentuée ». Prosôdia est dérivé de prosôdos «ce qu'on chante avec un instrument » et au fig. « ce qui s'accorde avec »)

Didact. 1. Caractères quantitatifs (durée...) et mélodiques des sons en tant qu'ils interviennent dans la poésie (surtout à propos du grec et du latin) Voir métrique, versification. les unités de la prosodie sont le mètre et le pied).

4 Rythme :

Alternance entre temps fort et temps faible, plein et vide, symétrie et dissymétrie, régularité et irrégularité, une alternance du même et du différent, mêlant indifféremment structure et périodicité ${ }^{1}$.

5 Ces trois définitions traditionnelles des notions de grammaire, prosodie et rythme, connues, intériorisées par tout lecteur lorsqu'il s'agit d'analyser un texte d'un point de vue linguistique, stylistique ou critique, ont en commun de donner la primauté à la quantité et à la régularité. Elles sont ainsi révélatrices de pratiques d'analyse qui séparent, par habitude et souci pédagogique sans doute, des aspects qui sont pourtant emmêlés dans l'acte d'énonciation, que l'énoncé produit soit considéré comme «poétique » ou comme « ordinaire ». Cependant, prises ensemble, dans leur interaction inévitable, elles rassemblent l'écrit (gramma signifie «lettre») - et donc le « lu»-, le 
parlé (intensité, timbre, accentuation) - soit aussi l'« entendu»-, le mouvement (balancement, structure et périodicité), - autrement dit le « senti ». Grammaire, prosodie, rythme renvoient ainsi, ensemble, aux caractéristiques externes, objectivables, de l'acte d'énonciation qui serait alors mû par une impulsion rythmique fondamentale transformée en geste expressif, parlé et/ou écrit.

C'est cette intuition qui est à l'origine de la formulation de notre hypothèse générale stipulant qu'au fondement de l'énonciation, il y a ce que nous désignons comme une opération de motivation rythmique dont toute l'organisation du discours s'ensuit et dépend. Le terme opération, entendu au sens culiolien d'enchaînement régulé d'opérations prédicatives et énonciatives, implique - c'est désormais bien connu - l'idée de relations et de traces de ces mises en relation, dans le cadre d'une linguistique qui considère qu'un métadiscours "séparateur ", commode à bien des égards, la détourne cependant de son objet premier tel que Culioli l'a défini (l'activité de langage à travers les langues naturelles et les textes) et du problème de cette linguistique-là qui est précisément d'« organiser les relations »:

J'ai été amené à montrer qu'il n'y avait pas de morphologie sans syntaxe, et pas de syntaxe sans morphologie, pas de syntaxe sans sémantique ni de sémantique sans syntaxe, donc que le problème était d'organiser les relations².

7 On pourrait " plagier » la formulation et dire qu'il n'y a pas de grammaire sans prosodie, pas de prosodie sans rythme ni de rythme sans prosodie, et que comprendre le rythme comme organisation du discours passe par la saisie des relations entre ces trois éléments. C'est donc le problème de leur interaction qu'une étude contrastive du poème d'Arthur Rimbaud, Le Bateau ivre, et des traductions de Samuel Beckett, Derek Mahon et Ciaran Carson nous permettra d'explorer.

Il s'agit en premier lieu de mieux préciser la notion de rythme dans les théories linguistiques puis d'observer comment une configuration récurrente dans le texte original $(j e+\mathrm{V})$ est traduite par des auteurs différents suivant l'interprétation propre à chacun des profils rythmiques du poème.

\section{La notion de rythme et les théories linguistiques}

9 La Théorie des Opérations Prédicatives et Énonciatives (TOPE) ne s'est que marginalement intéressée à la notion de rythme. Toutefois, aborder le fait linguistique dans sa totalité, comme le suggère la citation ci-dessus, est précisément l'une des raisons qui, dans ce cadre théorique, conduisent à s'interroger sur le caractère éventuellement opératoire de la notion et à s'en préoccuper afin d'en dégager l'importance. Par ailleurs, il serait inexact de dire que cet aspect du langage est totalement absent de l'élaboration théorique : de manière indirecte, la notion affleure à la surface du discours d'A. Culioli dans des textes anciens comme dans des écrits plus récents :

C'est que quand nous considérons un énoncé, nous tendons à l'intoner intérieurement, d'où des différences dont nous n'avons pas conscience, à le situer dans des contextes implicites qui entremêlent la plausibilité sémantique (ou pragmatique) et l'acceptabilité grammaticale ${ }^{3}$. (notre soulignement)

Sur la recherche, deux choses : d'abord j'ai lancé un certain nombre de recherches, sur des langues, dans des domaines où ce n'était pas fait, sur lesquels on ne s'interrogeait pas, la syntaxe, la prosodie ${ }^{4} .$. (notre soulignement) 
C'était l'époque [celle du structuralisme triomphant] où l'on vous disait: il y a la phonétique, la syntaxe, la sémantique - rien sur la prosodie d'ailleurs... La syntaxe ? en général, après la morphologie il n'y avait plus rien $[. . .]^{5}$ (notre soulignement) s'ajouter à la liste, comme un surplus, comme ce que, dans l'analyse, on oublie souvent, laisse penser que la théorisation du rythme en termes d'opérations est encore à faire, puisqu'elle se cantonne dans le fait prosodique. Culioli n'emploie d'ailleurs pas le mot rythme, il lui substitue justement celui de prosodie ou parfois celui d'intonation sans que soit clairement établie de distinction entre ces deux derniers termes, ou bien encore, en de plus rares occurrences, celui de mouvement ${ }^{6}$. À ce propos, on pourrait suggérer que la quasi absence de cette notion en tant que telle dans les textes de Culioli vient peut-être du développement, à la même époque, d'une autre théorie du langage, celle d'Henri Meschonnic, et de la nécessité de s'en démarquer puisqu'à l'ancrage, à la fois formel et « réaliste» de l'une (son caractère "scientifique ») s'oppose l'utopie de l'autre (d'une certaine façon sa démesure). Renvoyés du côté de la littérature, les travaux de Meschonnic qui s'appuie, lui aussi, sur une critique du signe, constitueront la seconde référence à l'arrière-plan de notre tentative.

11 Cette entreprise théorique ne s'est jamais présentée comme une linguistique, ni même comme une théorie de la littérature et, en raison de cela, elle reste suspecte aux yeux des linguistes de tout bord comme à ceux des littéraires. Refusant de considérer séparément la littérature (« le poème ») et le langage, elle s'est donnée d'emblée comme une théorie du langage, une théorie inclusive donc, englobant linguistique, littérature et traduction, faisant même de la traduction, ou plus exactement, $d u$ «traduire » le paradigme de son éthique. La traduction de la Bible et le statut du rythme dans la Bible sont pour Meschonnic la source même de sa critique. Les résultats n'en sont pas moins généralisables :

Mais l'exemple de la Bible, particulier qu'il est et même unique, ne fait que porter à

la «dénudation» du procédé, comme disaient les formalistes russes, un fait empirique général, qui est la place du rythme dans une physique du langage ${ }^{7}$.

Nous retenons de cette critique théorique trois éléments : (i) l'idée de continu, qui n'exclut ni le discontinu, ni l'existence de «niveaux » d'analyse du langage. L'enjeu est davantage de donner un statut théorique à l'existence empirique du continu que de nier le discontinu car il y a dans le langage du continu et du discontinu mais ce dernier n'est pas à côté du continu, il est pris dedans ; (ii) l'imbrication du rythme et de la signification constituant ensemble la "signifiance». Ils ne peuvent en effet être dissociés dans la mesure où le rythme est un principe organisateur; et (iii) la possibilité que cette entreprise ouvre d'une invention d'une "grammaire du rythme qui rende compte de l'activité de langage que sont les discours, à partir d'autres catégories que celles, traditionnelles, de la langue ${ }^{8} »$. Dans la perspective de Meschonnic et Dessons, et la définition large qu'elle suppose, le rythme, loin d'être une notion formaliste, opère la synthèse de ce qui est en jeu dans l'acte d'énonciation:

On a parfois assimilé les études de rythme (dans leur réception, comme dans leur émission) à un ensemble d'opérations purement formelles, une sorte d'arithmétique accentuelle sans rapport avec la signification des textes. Or, s'il est légitime de qualifier le rythme de "formel ", dans la mesure où, organisant les discours, il leur donne littéralement forme, le rythme n'est pas formaliste, au sens où il n'est pas une forme vide, un ensemble schématique qu'il s'agirait de montrer ou non, selon l'humeur. Le rythme en est l'élément fondamental, puisqu'il n'est 
rythme que d'opérer la synthèse de la syntaxe, de la prosodie et des divers mouvements énonciatifs de ce texte. n'est pas une forme vide, un ensemble schématique ») une critique à peine voilée de la perspective culiolienne, ou plutôt de ce qui a été, et peut-être demeure, un objectif, à savoir, entre autres choses, la formalisation du langage et la réduction de configurations " prosodico-syntactico-sémantico-pragmatiques ${ }^{9}$ » à des formes schématiques. Dans la mesure où toute théorie construit sa propre cohérence, on pourrait objecter le risque d'amalgame et la difficulté de s'accommoder de la différence de point de vue que ces deux constructions théoriques manifestent. À vrai dire, c'est l'exploration elle-même qui permettra de répondre à cette objection et, si l'on se réfère à Benveniste, l'idée du rythme comme principe organisateur de tout ce qui est mouvement ne saurait être étrangère à une théorisation de l'énonciation. S'y manifeste, en effet, une activité énonciative qui est celle d'un énonciateur-coénonciateur (selon Culioli), ou celle d'un sujet (selon Benveniste et Meschonnic ${ }^{10}$ : dans, et à travers l'acte d'énonciation, se construit une subjectivité qui n'est que mouvement et rythme. d'un côté à un concept épistémologique (« l'organisation du mouvant»), de l'autre à un « marquage » qui est l'inscription d'un sujet-énonciateur dans son discours :

Il importe [...] de noter que le rythme, comme notion propre à l'épistémologie des «sciences» du langage, et irréductible au modèle du signe, et le rythme comme marquage des moments, des temps forts du discours, sont distincts. Cependant, le dernier point de vue n'est que l'épreuve et la vérification du premier: tous deux ensemble sont une seule et même écoute du langage. Sans quoi la notation du rythme retomberait dans la notion traditionnelle, formelle, et l'entreprise tout entière en redeviendrait dénuée de sens.

Très concrètement, s'approprier le rythme d'un texte, s'y glisser, mais surtout l'analyser revient à interpréter ses marques, à différents niveaux. Pour le texte poétique, il s'agira notamment de retrouver ce que la tradition a déjà répertorié, métrique, rimes et autres principes de versification, prosodie, accents et intonation afin de saisir le mouvement énonciatif et l'opération rythmique dont ils sont la trace.

\section{Considérations pratiques}

Il apparait d'ores et déjà que l'un des premiers problèmes est, sinon de renouveler la notation du rythme, de s'appuyer sur une notation homogène et cohérente pour que la seule description des marques des «temps forts du discours » ne soit plus une fin en ellemême mais qu'au contraire, ces marques soient bien la trace liant l'énoncé à l'énonciation, autrement dit aux opérations de construction de l'énoncé rythmé. Il importe que cette notation ne soit pas seulement un codage mais devienne une clé pour reconstruire les relations produisant la signifiance du discours. Dans le domaine français, nous retiendrons la proposition de Dessons et Meschonnic (Traité du rythme, 1998) et, pour l'anglais, celle de Derek Attridge (Poetic Rhythm, 1998). Ce dernier sépare poésie et prose et, progressivement, passe d'une notation accentuelle modulée à la mise en place d'une représentation de ce qu'il appelle phrasing, le phrasé, qui intègre la syntaxe et la signification : 
In our discussion of English verse forms in the foregoing chapters, we have frequently noticed the important part played by syntax and meaning in the movement of poetry. [...]

We call this dimension of the movement of poetry phrasing, a word which refers both to syntax and to meaning, and which in its form ("phrasing" rather than "phrases") indicates the dynamic nature of the phenomenon it refers to. [...]

A poem's phrasing is an important part of its varying sense of pace and onward impetus, and of its different degrees and types of pause and closure. Like meter and rhythm, phrasing is not something we add to language but something that is already part of what we know and do when we speak and understand a language ${ }^{11}$.

On voit bien que les concepts ne se superposent pas exactement d'une langue à l'autre puisque pour Attridge, rhythm est différent de phrasing alors que pour Meschonnic et Dessons, la notion de rythme comprend le phrasé : il est ce que le sujet fait lorsqu'il parle. L'approche d'Attridge réhabilite les rythmes de la langue parlée ("Poems are made of spoken language") ; elle est descriptive, pratique et technique :

Rhythm is a patterning of energy simultaneously produced and perceived; a series of alternations of build up and release, movement and counter-movement, tending towards regularity but complicated by constant variations and local inflections.

Toutefois, en dépit de ces différences, ces deux approches empiriques se rejoignent dans l'idée d'une "physique du langage " et constituent, à ce tout premier stade de notre investigation, des mises en ordre et des repères concernant notamment la terminologie, les problèmes de scansion et la notation.

Replaçant tout cela dans le cadre d'une linguistique contrastive, résumant ce qui précède et anticipant sur les problèmes que posera la comparaison d'un original et de ses traductions, nous pouvons suggérer :

- que le rythme est en premier lieu une expérience, celle d'une énergie énonciative qui fait de l'énonciation même un mouvement continu,

- qu'il se réalise dans la langue tout en construisant par la synthèse de la prosodie et de la syntaxe une signification du discours qu'il impulse,

- que lors du passage d'un système linguistique à l'autre, la question se pose de savoir si l'énonciation traductive intègre, plus ou moins consciemment, des phénomènes d'ordre rythmique récurrents, des rhythmic patterns ou configurations rythmiques, qui appartiendraient à ce que Jacqueline Guillemin-Flescher appelle «l'organisation collective du discours ». Elle-même invite à intégrer cette notion de rythme dans la réflexion théorique sur les processus de traduction lorsqu'elle écrit :

Je proposerais comme hypothèse que plus la qualité rythmique d'un texte est marquée plus on pourra aller loin dans le respect de l'original. Ce n'est pas un hasard si la Bible et Shakespeare sont souvent évoqués dans la théorie de la traduction car ils constituent en cela des exemples privilégiés ${ }^{12}$.

Cette hypothèse ne semble pas avoir été par la suite examinée dans toutes ses implications. Elle n'est pas sans poser de nombreuses questions d'ordre théorique qui débordent le propos de cet article mais elle précise l'enjeu de cette tentative qui est de 
donner forme à « la qualité de la quantité » ce qui, sans doute, ne se révèle pleinement que dans le contraste des langues.

\section{Profils rythmiques du Bateau ivre}

21 Le profil rythmique du poème de Rimbaud, en tant que poème-texte, se caractérise par la rencontre, sinon le conflit, entre ce que Meschonnic appellerait du « continu sémiotique » (expression qui renvoie au régime des signes, la forme et le contenu constituant une polarité sémiotique) et le «continu comme rythme » (expression qui renvoie à l'idée du " corps dans le langage ", c'est-à-dire à l'activité de langage faite d'affect, de geste, de mouvement, de parole $)^{13}$. Cette rencontre est celle de la langue et du discours : de cette rencontre naît le rythme comme organisation du sens.

22 À l'évidence, la longueur du poème (vingt-cinq strophes) est le premier de ses traits marquants. S'y ajoutent la régularité des strophes (quatrains rimés abab) et l'unité métrique, qui est encore l'alexandrin. Ces trois caractéristiques prises ensemble se conjuguent pour suggérer, créer même, un mouvement. La lecture à haute voix confirme ce que l'œil a aperçu : de strophe en strophe, le discours gagne à la fois en vitesse et en intensité par un effet d'entraînement difficile à stopper et pourtant, empêché : l'énonciateur-lecteur, une fois embarqué, bute à partir de la huitième strophe sur la récurrence d'un schéma prédicatif centré sur la première personne pronominale, double repère (prédicatif et constitutif) d'une relation cruciale au cœur du poème. C'est un peu comme si le lecteur était entraîné dans un mouvement giratoire violent, s'amplifiant jusqu'à atteindre un paroxysme (à la strophe 14) pour retomber dans une accalmie, avant de reprendre de l'énergie jusqu'à la vingt et unième strophe dont le dernier vers ( $\mathrm{Je}$ regrette l'Europe aux anciens parapets ») annonce la dépression des quatre dernières. Ce mouvement contraire, comme empêché, est clairement manifesté par une dérégularisation de l'alexandrin: la césure est généralement à l'hémistiche dans les premières strophes («Comme je descendais des fleuves impassibles» [1], «J'étais insoucieux de tous les équipages » [2]) mais elle est décalée vers le début du vers ou bien vers la fin, à partir de la récurrence du schéma «NP + GV/Je... » qui, si elle s'inscrivait dans un rythme immuable, perdrait la force que lui donne un rythme déstabilisé, et par conséquent surprenant ( Je sais les cieux crevant en éclairs, et les trombes/Et les ressacs et les courants [...] » [8]/«J'ai suivi, des mois pleins, pareille aux vacheries/Hystériques, la houle à l'assaut des récifs » [11]/« Mais, vrai, j'ai trop pleuré! Les Aubes sont navrantes. » [23]). Et, presque à chaque fois, le rythme de l'alexandrin ainsi déstabilisé, requiert un enjambement pour récupérer le continu du discours.

Ce profil est interprété différemment par les trois traducteurs, ce que l'aspect visuel de chaque traduction manifeste. Celle de Beckett (1976) conserve le même nombre de strophes, reproduit, imite et se caractérise par la régularité, le calque et une homogénéisation qui réduisent la part de désorientation inhérente au rythme de l'original. L'impétuosité est comme atténuée. La seconde, chronologiquement, celle de Derek Mahon (1985), frappe par sa régularité mais surtout par sa condensation. Tout ce qui contribue à la perte de repères est effacé : elle ne compte que douze strophes au lieu de vingt-cinq et, sémantiquement, s'en tient au minimum, à savoir ce qui dans l'original se ramène à l'idée d'une vie en poésie. L'accumulation des visions est élaguée. La dernière, celle de Ciaran Carson (1993), garde le même nombre de strophes mais travaille le mouvement, à l'oreille et visuellement. Carson choisit un vers long, souple, favorisant 
la variation et, surtout, la continuation d'un vers à l'autre par l'enjambement. Le second vers est plus court, et le schéma est répété pour les deux derniers vers de chaque quatrain, ce qui réintroduit la régularité d'ensemble. L'absence délibérée de la régularité de l'alexandrin est donc ici interprétée et transposée dans un autre schéma métrique qui fait la part belle à l'enjambement que nous retiendrons comme étant ici le procédé technique le plus récurrent pour traduire le «continu» du rythme. Carson s'écarte du pentamètre iambique classique pour faire correspondre à l'alexandrin "moderne » (ou "fin d'époque ») de Rimbaud, un vers dont le rythme est plus proche de celui de la ballade, faisant rimer le second et le quatrième vers.

Les différences marquées entre ces trois réalisations par la traduction du profil apparent du poème pourraient amener à porter un jugement critique en faveur de la traduction de Carson, plus inventive, dans sa forme, que celle de Beckett, plus respectueuse de la totalité du poème que celle de Mahon. Or, la traduction de Mahon fait apparaître un autre profil rythmique sous-jacent, une sorte de matrice à partir de laquelle la double notion d'ivresse et de désenchantement s'est développée : le bateau est entraîné dans l'ivresse d'une navigation libre par la violence d'une rupture ratifiée par des sacrifices humains (première strophe) et rendu à sa petitesse lorsqu'il se métamorphose en frêle bateau de papier fabriqué par un enfant (vingt-quatrième strophe).

\section{La construction du « je »}

25 Le jeu induit par le glissement d'un profil rythmique chaotique à ce profil essentiel, au sens où il renvoie au poème dans le poème, est à l'origine d'une tension qui invite à reconsidérer l'identification simple, presque trop évidente du poète et du bateau : je est le bateau ivre est le poète... Le bateau est aussi, et tout autant, dans une relation de différenciation avec le poète. C'est un objet, un petit jouet, et l'accès pérenne de l'enfantpoète $^{14}$ à la voyance, dont pourtant il jouit un moment, est démenti par l'image - sinon la présence - rémanente de l'enfant-enfant rendu au monde triste qu'il n'ose pas, ou ne peut, quitter encore. Ce sont bien là deux rythmes qui entrent en conflit l'un avec l'autre jusqu'à rendre la poésie à venir impossible parce qu'un « je », qui ne serait pas seulement " un autre » mais aussi « soi-même », échoue à se construire.

Cette irrésolution d'un je pourtant omniprésent est construite dans le rythme même de ses occurrences, prosodiquement et syntaxiquement, ainsi que dans les mouvements énonciatifs, contradictoires ou non, du texte. L'enjeu est alors de donner de la force à la forme inaccentuée du pronom personnel je pour qu'apparaissent les deux faces irréconciliables de son référent.

Si la récurrence du pronom est un élément non négligeable dans la perception de cet élan qui pousse un sujet «je » à se manifester, ce n'est cependant pas tant le nombre d'occurrences qui compte ${ }^{15}$ que leur distribution dans le poème tout entier et dans les vers où ils se trouvent. D'un point de vue syntaxique, la structure dans laquelle s'insère le pronom ne présente rien de particulier. Le pronom est à la fois sujet grammatical, repère et repère constitutif de la relation prédicative et énonciative et, dans la première partie du poème (strophes 1 à 8), tout comme dans les deux dernières strophes (24 et 25), le pronom je, garde son caractère atone, au sens phonétique d'inaccentué. Il est aussi " atonique »: le sujet qui s'énonce dans ces deux parties inégales ne s'affirme pas comme un sujet « agent » ou « acteur » du monde mais comme un sujet sans volonté propre et, à la toute fin du poème, comme un sujet résigné. En attestent les verbes de perception ( $/ \mathrm{Je}$ 
ne me sentis plus guidé », vers 2 , strophe 1), les prédications de propriétés («j'étais insoucieux de tous les équipages", vers 1 , strophe 2), la forme pronominale ( «Je me suis baigné dans le Poème/De la Mer », vers 1 et 2, strophe 6), la forme hypothétique de «Si je désire une eau d'Europe... » (vers 1, strophe 24) et la forme négative (« Je ne puis plus... ») de la dernière strophe du poème. Emporté donc par le rythme euphorique d'une navigation au gré des fleuves et de la mer, il se déprend de tout lien et s'abandonne à l'ivresse du "Poème de la mer » (strophe 6) - avant de basculer dans l'expérience de la voyance (toute la partie médiane du poème) - pour enfin revenir à la réalité. Les deux dernières strophes expriment le désenchantement, la dés-illusion, et la nostalgie de l'enfance.

C'est donc l'expérience de la voyance qui transforme un «je " à la dérive en un sujet halluciné qui s'affirme dans la longue partie médiane du poème (strophe 9 à 22). C'est là que s'opère le passage de la forme atone du pronom à son expression accentuée. Le son [3] devient un phonème accentué lorsqu'il se combine avec le son vocalique [e]. Ce glissement de [3ə] à [3e] est amorcé et réalisé dans la strophe $8:$ : je sais » ([3əse]) est répété deux fois (vers 1 et 2) et se transforme en «j'ai vu» ([3evy]) au début du dernier vers: «Et j'ai vu quelquefois ce que l'homme a cru voir!» Vision, expérience et connaissance donnent consistance au sujet. Celui qui dit « je » n'existe plus que dans la force de ce rythme d'attaque au début de chacune des cinq strophes suivantes ( J'ai vu», "J'ai rêvé ", "J'ai suivi ", "J'ai heurté ", "J'ai vu »), rythme qui fait la synthèse d'une position de sujet grammatical, d'une position stratégique en début de vers et de strophe, et de la forme verbale aspectuelle de l'accompli. Ce « je » devient ensuite « moi », la forme véritablement accentuée du pronom sujet mais en se dédoublant de « je » à « moi », il ne rencontrera que lui-même, sans jamais trouver l'autre, ce «tu » qui ferait de lui un être engagé dans le langage du poème. Le seul "tu » évoqué est encore son double qui lui échappe de toute façon : «- Est-ce en ces nuits sans fonds que tu dors et t'exiles,/Million d'oiseaux d'or, ô future Vigueur? » (vers 3, strophe 22).

Au-delà de l'évidente traduction du pronom je par le pronom I, est-il possible de retrouver dans les trois traductions examinées ici ce jeu prosodique qui met en lumière le double dédoublement d'un sujet d'abord pris par le poème, puis voyant, puis critique?

Suivant l'interprétation par chaque traducteur du profil rythmique du poème tout entier, chacune des traductions construit un sujet inscrit dans ce même rythme. La traduction de Beckett frappe par une quasi conformité à l'original mais également par une utilisation de la langue anglaise qui ne réserve aucune surprise : des déplacements du syntagme verbal (sujet + verbe) sont opérés dans les premières strophes pour retrouver une sorte de logique prédicative propre à l'anglais comme, par exemple, dans la troisième strophe :

\begin{tabular}{|l|l|}
\hline Dans les clapotements furieux des marées, & Blanker than the brain of a child I fled \\
\hline $\begin{array}{l}\text { Moi, l'autre hiver, plus sourd que les cerveaux } \\
\text { d'enfants, }\end{array}$ & $\begin{array}{l}\text { Through winter, I scoured the furious jolts of } \\
\text { the tides }\end{array}$ \\
\hline Je courus ! Et les péninsules démarrées & In an uproar and a chaos of Peninsulas, \\
\hline N'ont pas subi tohu-bohus plus triomphants. & Exultant, from their moorings in triumph torn. \\
\hline
\end{tabular}

31 L'inversion des deux premiers vers rétablit une chronologie qui annule l'effet de précipitation et d'exaltation de l'original même si la position de I fled en fin de vers 
signale une mise en relief comparable à la position de Je courus au début du troisième vers. De même l'ajout d'une autre prédication permettant la répétition du pronom I a sans doute pour effet de compenser l'absence en anglais de deux formes de pronom sujet, la forme moi assurant en français sans redondance la prééminence du sujet je dans cette strophe. Dans cette même strophe, Mahon efface les pronoms pour évoquer le sujet au moyen d'une métonymie : "my heart rose to a tidal detonation » (vers 2) tandis que Carson révèle l'enjeu poétique de l'expérience à venir, qui n'est autre que la maîtrise du langage, en introduisant une série d'assonances et d'allitérations sur des verbes dont le choix relève d'une interprétation totalement libre :

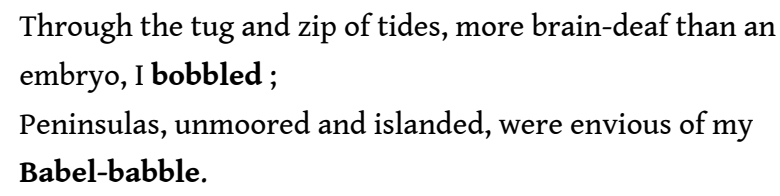

Il n'y a donc pas ici de jeu prosodique sur le pronom lui-même qui garde sa forme inaccentuée puisque c'est la forme verbale, et le nom, qui reçoivent l'accent sur la première syllabe.

Si les traductions sont, pour cette strophe, très différentes l'une de l'autre, ce n'est pas le cas dans la traduction de la forme pronominale («je me suis baigné dans le Poème/de la Mer », strophe 6) pour laquelle les trois traducteurs adoptent, naturellement, une forme passive (participe passé ou diathèse passive) :

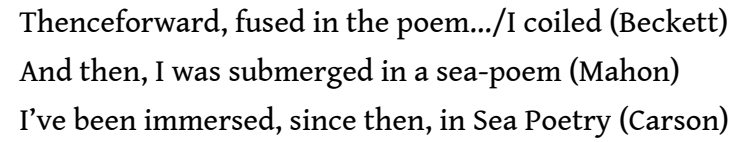

Plus que de traduction, c'est de transposition qu'il s'agit, au sens de Jakobson, dans un rythme lié d'une part à l'opération de prédication qui privilégie le verbe et d'autre part au choix énonciatif d'un discours de type narratif qui raconte plus qu'il ne voit. Là encore, c'est le verbe qui est prosodiquement mis en lumière.

La détermination verbale indique alors le mode de relation entre le sujet et le prédicat et a donc une incidence sur le degré d'accentuation du pronom I. Dans la partie médiane du poème, celle où en français le sujet s'accomplit comme "voyant", Beckett conserve, comme on peut s'y attendre, la forme auxiliée de l'accompli sans contracter l'auxiliaire, ce qui ne neutralise le pronom qu'à demi : ce qui est perçu l'est par rapport à ce point de vue («I have seen », "I have dreamt », «I have followed ", «I have fouled », "I have seen » à l'initiale du premier vers des strophes 9 à 13). En revanche, dans la traduction élaguée de Mahon, le sujet I est l'origine d'une perception qui ressortit, dans la logique narrative de Mahon, à un événement ("I saw ", «I dreamed ", "I heard », à l'initiale des strophes 7,9 et 10). Lorsque cette perception est celle d'une origine active, le sujet grammatical I est omis (医 watched). Plus que le sujet lui-même, c'est bien l'événement qui compte de telle sorte qu'il impose son rythme au sujet. De façon plus complexe, Carson joue sur le registre d'une narration orale et alterne la forme aspectuelle de l'accompli, en utilisant la forme contractée de l'oral, et la forme simple du passé renvoyant à une occurrence d'événement: «I've known", «I've seen", strophes 8 et 9 , mais «I dreamed », «I followed ", «I bumped », « I saw », strophes 10 à 13, et de nouveau «I've drifted off », « I've whinged », strophes 21 et 23. Ce relevé non exhaustif montre les 
variations rythmiques possibles internes à la traduction selon la perception et l'interprétation par le traducteur du rôle tenu par le sujet et de sa relation avec le prédicat. Il est clair que le rythme des configurations verbales I have seen, I've seen, et I saw n'est pas le même mais il est difficile, en dépit de cette différence, de mesurer le degré d'accentuation du pronom, degré qui dépend aussi d'autres éléments du contexte et de la lecture que chaque lecteur en fait.

L'ambivalence chez Beckett est sans doute la marque d'une volonté de traduire l'original au plus près. La simplification chez Mahon est peut-être, paradoxalement, la trace d'une identification au poète-énonciateur. Chez Carson enfin, l'alternance des formes simples et des formes auxiliées contractées relève d'une recherche de connivence et de familiarité ; elle construit le sujet I dans le rythme de l'oral et d'une coénonciation plus affirmée. Elle risque l'accentuation là où les autres ne s'écartent pas de l'ordinaire de la langue, comme dans la juxtaposition des pronoms me et $I$ : « Me, I shivered: fifty leagues away, I heard the bumbling/Behemoths and Scarabs" (strophe 21) ${ }^{16}$. Tous cependant reviennent à une présence défaite du sujet dans les deux dernières strophes, en conformité avec l'original.

L'impact rythmique du pronom personnel, qui n'est donc normalement pas accentué, mais que l'on peut promouvoir au statut de phonème accentué dans certaines conditions, est ainsi déterminant dans la construction de la signifiance du poème et détermine plus ou moins consciemment la lecture qu'on en fait. La façon dont la traduction rend compte de cet impact influe à son tour sur la compréhension du poème par un lecteur étranger mais l'évidence d'une correspondance terme à terme entre le pronom français je et l'anglais $I$ en masque la portée. Les traductions mettent surtout en évidence la singularité de la lecture et de l'interprétation. En effet, les ruptures dans la construction de ce sujet apparaissent moins dramatiques, et donc moins visibles, dans les traductions que dans l'original car on n'y observe pas ce passage d'une forme atone à une forme plus accentuée lorsque s'opère la mutation du poète en voyant, condition nécessaire pour rester Poète, inspiré et critique, pour devenir «le suprême Savant»: "Car il arrive à l'inconnu! Puisqu'il a cultivé son âme plus qu'aucun! Il arrive à l'inconnu, et quand, affolé, il finirait par perdre l'intelligence de ses visions, il les a vues ${ }^{17}$ ! »

À partir de l'interprétation différente du profil rythmique du poème, chaque traducteur a développé une dimension rythmique propre qui n'est plus le rythme de Rimbaud. La traduction de Beckett, la plus proche de l'original, montre l'impossibilité d'une traduction du rythme car l'anglais ne trouve pas son rythme. Celle de Mahon ne rend pas le conflit interne au sujet qui s'énonce ni les souffrances qui accompagnent l'expérience de la voyance. Enfin, le travail de Carson sur le mètre, les marqueurs du rythme que sont l'enjambement, les allitérations et assonances, et la forme du poème laisse penser que des correspondances ne sont pas impossibles d'une langue à l'autre.

\section{NOTES}

1. Adapté du Dictionnaire culturel en langue française, sous la direction d'Alain Rey, Paris, Dictionnaires Le Robert, 2005. 
2. Antoine Culioli et Claudine Normand, Onze rencontres sur le langage et les langues, Paris/ Gap, Ophrys, 2005, p. 56.

3. Antoine Culioli, «La linguistique: de l'empirique au formel» [1987], Pour une linguistique de l'énonciation. Opérations et représentations, t. 1, Gap, Ophrys, 1990, p. 9-46 (17).

4. Culioli/Normand, op. cit., p. 53.

5. Ibid., p. 57.

6. Ce qui s'accorde d'ailleurs avec l'idée d'ajustement et de déformabilité des représentations linguistiques (voir sur ce point Antoine Culioli, Pour une linguistique de l'énonciation, t. 2, Paris/Gap, Ophrys, 1999, p. 45).

7. Henri Meschonnic, « Rythme et langage », Dictionnaire Culturel en Langue Française, sous la direction d'Alain Rey, Dictionnaires Le Robert, 2005, p. 465-469 (466).

8. Gérard Dessons et Henri Meschonnic, Traité du rythme, des vers et des proses, Paris, Dunod, 1998, p. 7 et, pour les deux citations suivantes, op. cit., p. 6 et p. 74-75.

9. Voir Culioli/Normand, op. cit., p. 95.

10. Il serait cependant imprudent de superposer et d'assimiler trop vite les concepts d'énonciateur-coénonciateur et de sujet qui correspondent à deux façons d'envisager la subjectivité à l'œuvre dans le langage. Par commodité, nous parlerons de sujeténonciateur.

11. Derek Attridge, Poetic Rhythm. An Introduction, Cambridge, Cambridge University Press, [1995] 1998, p. 182, et p. 3 pour les deux citations suivantes.

12. Jacqueline Guillemin-Flescher, "Un linguiste devant la traduction», Fabula, $n^{\circ} 7$, Traduire, Lille, Presses Universitaires de Lille, 1986, p. 59-68 (66).

13. Voir Henri Meschonnic, « La Force dans le langage », Chiss, J.-L. et Dessons, G., La Force du langage. Rythme, Discours, Traduction. Autour de l'cuvre d'Henri Meschonnic, Paris, Honoré Champion, 2006, p. 16.

14. On se souvient que Rimbaud n'a que 17 ans lorsqu'il écrit Le Bateau ivre au cours de l'été 1871.

15. Vingt-quatre occurrences du pronom je, six occurrences du pronom moi dont une seulement en position complément, et dix occurrences de mon, me, et mes.

16. «Moi qui tremblais, sentant geindre à cinquante lieues/Le rut des Béhémots et les Maelstroms épais ».

17. Rimbaud, Poésies complètes, 1870-1872, Paris, Le Livre de Poche, LGF, 1998, p. 151.

\section{INDEX}

Mots-clés : poésie - prosodie, Carson Ciaran, Rimbaud Arthur, traduction, relations francoirlandaises, Mahon Derek

Keywords : poetry - prosody, translation, Franco-Irish relations 


\section{AUTEUR}

MARYVONNE BOISSEAU

Université de Strasbourg 